\title{
Study of moxibustion stimulation in the ovariectomized rat model
}

\author{
Shigeyuki Kanai ${ }^{1, *}$, Norimasa Taniguchi ${ }^{1.2}$, Kayo Kanda ${ }^{1}$ and Izuru Matsuhata ${ }^{3}$ \\ 'Kansai College of Oriental Medicine, 2-11-1 Wakaba Kumatori-cho, Sennan-gun, Osaka 590-0482, Japan; \\ 'Department of Science PIP-FUJIMOTO Co,Ltd, 1-36 Noninbashi 2-choume, Chuo-ku, Osaka 540-0011, Japan; \\ ${ }^{3}$ Pain Clinic, Department of Anesthesiology, Osaka Medical College, 2-7Daigakutyo, Takatuki-shi, Osaka -fu 569- \\ 8686, Japan
}

\begin{abstract}
SUMMARY
In order to examine the effectiveness of moxibustion stimulation for climacteric disturbance, we administered moxibustion stimulation to ovariectomized (OVX) rats, and compared the tail surface temperature, laboratory parameters, and the level of locomotor activity with those of untreated rats. Twenty-four female Wistar rats ( 8 weeks old, $160-180$ g) were divided into three groups. The rats in the OVX-M group underwent moxibustion stimulation after ovariectomy. The rats in the OVX-C group underwent ovariectomy but did not receive moxibustion stimulation. The rats in the Normal group received neither ovariectomy nor moxibustion stimulation. The level of locomotor activity was determined by a metabolism measuring system. The tail surface temperature was significantly lower in the OVX rats before moxibustion stimulation than in the Normal group. In the OVX rats before moxibustion stimulation, there was no clear difference in the level of locomotor activity between the active and resting phases, and the pattern of locomotor activity was irregular. After moxibustion stimulation, the tail surface temperature of the OVX-M group did not significantly differ from that of the Normal group, and the pattern of locomotor activity of the OVX-M group became diphasic with clear active and resting phases, similar to that observed in the Normal group. These results demonstrate that moxibustion stimulation is effective for the treatment of climacteric disturbance.
\end{abstract}

Key words: Moxibustion; Ovariectomized rat; Thermography; Locomotor activity

\section{INTRODUCTION}

The biological responses to moxibustion have been widely studied from the perspective of possible health effects. It has been reported that moxibustion stimulation has beneficial effects on chronic complaints. Moxibustion stimulation has been used to treat insomnia and headache, as well as to provide relief from pain due to ischemia in

*Correspondence: Shigeyuki Kanai, Kansai College of Oriental Medicine, 2-11-1 Wakaba Kumatori-cho, Sennan-gun, Osaka 590-0482, Japan. Tel: +81-724-538251; Fax: +81-724-53-0276; E-mail: kanai@kansai.ac.jp the blood microcirculation (Chen, 1987; Uchida et al., 2003). After menopause or ovariectomy, women tend to develop menopausal symptoms including hot flashes, abnormal feelings, palpitations, and insomnia (Harada and Nakata, 1995). In particular, premenopausal women who have undergone ovariectomy develop osteoporosis and severe menopausal symptoms due to sudden estrogen deficiency. Many studies have recently reported that moxibustion stimulation reduced the number of complaints of postmenopausal women (Zhao et al., 2000; Zhang et al., 2004). In the present study, we investigated whether ovariectomized rats can 
serve as an animal model of climacteric disturbance and whether moxibustion stimulations reduce the symptoms of climacteric disturbance.

\section{MATERIALS AND METHODS}

\section{Animals and environmental conditions}

This study was approved by the Animal Committee of Kansai College. Female Wistar rats (age, 7 weeks; body weight, about $150 \mathrm{~g}$ ) were purchased from Japan Crea Co., Ltd. (Shizuoka, Japan). The animals were housed individually in cages and kept in a room that was maintained at a temperature of $23 \pm 1^{\circ} \mathrm{C}$ with a relative humidity (RH) of $55 \pm 5 \%$ under a $12 \mathrm{~h} / 12 \mathrm{~h}$ light-dark cycle (light: 9:00AM to 9:00PM). Solid rodent chow and tap water were given ad libitum. After 1 week of acclimation under these conditions, animals showing favorable growth were selected and used for further studies.

\section{Experimental groups}

The twenty-four rats aged 8 weeks were divided into 3 groups of 8 rats each. Bilateral ovariectomy was performed in the rats in the OVX-M and OVX$C$ groups under anesthesia with ether. Moxibustion stimulation was administered to the rats in the OVX-M group. The rats in the OVX-C group were not treated with moxibustion stimulation after ovariectomy and they served as a control group. The rats in the Normal group underwent neither ovariectomy nor moxibustion stimulation, and they served as the normal control group.

\section{Experimental schedule}

After a postoperative recovery period of 3 weeks, moxibustion stimulation was started in the OVX$M$ rats. Moxibustion stimulation was performed under ether anesthesia at 2 sessions per week for a total of 8 sessions. Moxibustion stimulation was performed on days $0,4,7,11,14,18,21$ and 25 . The rats in the OVX-C and Normal groups were anesthetized with ether in a similar manner 2 times per week for a total of 8 times, but they did not receive moxibustion stimulation.

In the OVX-M group, prior to moxibustion stimulation, the rat was mildly anesthetized with ether. During a moxibustion stimulation session, moxibustion was repeated 3 times by placing a moxa ball of half-rice grain size onto the Guanyuan $\left[\left(\mathrm{CV}_{4}\right) 5 \mathrm{~mm}\right.$ below umbilicus] and Sanyinjiao [(SP6) $3 \mathrm{~mm}$ upper medial malleolous of tibia posterior] for 10 min. The sites to be stimulated by moxibustion were determined by consulting the rat meridian points defined by Jo bon N (2003).

Measurement of body weight and serum total cholesterol (T-chol) level before and after moxibustion stimulation for 4 weeks

The body weight and serum T-chol level were measured before and after 4 weeks of moxibustion stimulation on days 0 and 28. A blood sample (1.5 $\mathrm{ml}$ ) was collected from the cervical vein under ether anesthesia, and the serum T-chol level was measured using the Wako Serum Test Kit (Wako Pure Chemicals Industry, Osaka, Japan).

Measurement of tail surface temperature before and after moxibustion stimulation for 4 weeks

To avoid the influence of the haircoat, the tail surface temperature which is used as an indicator of peripheral circulation, was measured. The tail surface temperature was measured using a thermograph (TVS-2300 MkIIST, Japan Abionics Co., Ltd., Tokyo, Japan) before and after 4 weeks of moxibustion stimulation on days 0 and 28 . After 15 min of acclimation to the environment, the tail surface temperature was measured in conscious animals in a windless room maintained at a temperature of $15 \pm 1^{\circ} \mathrm{C}$ and a RH of $55 \pm 5 \%$. The temperature of the rat tails was measured at a distance of $1 \mathrm{~m}$ from the thermography device.

Measurement of locomotor activity before and after moxibustion stimulation for 4 weeks

Locomotor activity was measured using a 


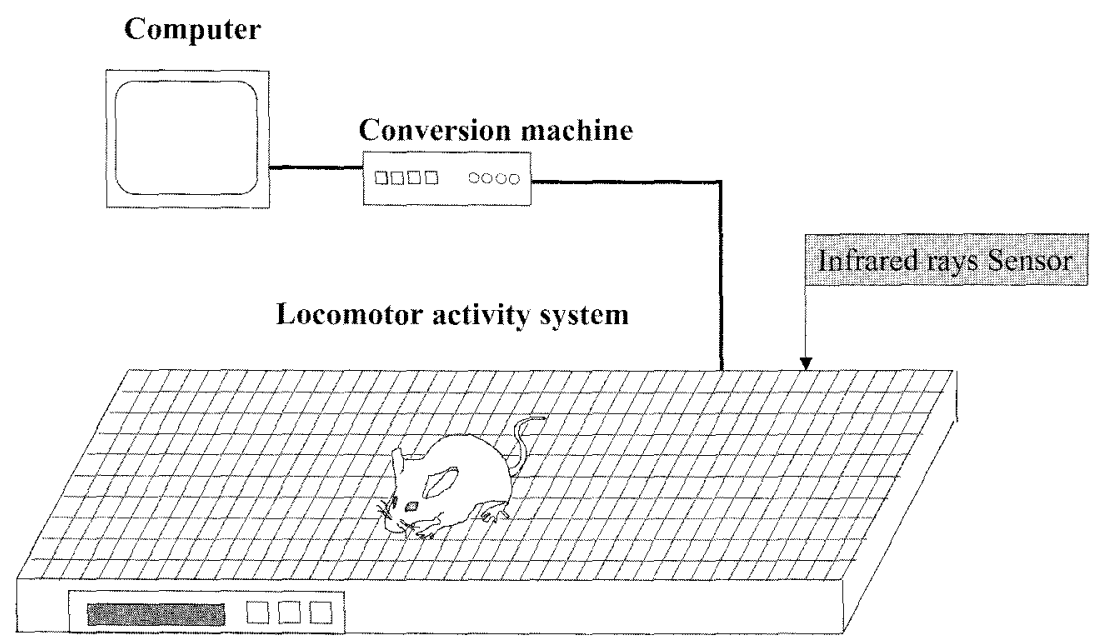

Fig. 1. Diagram of the metabolism measuring system (SCANET MV-10; MELQUEST Co., Ltd., Toyama, Japan) that was used to measure the level of locomotor activity of a rat.

metabolism measuring system over a 24-hour period ( 1 day) before starting moxibustion stimulation and after moxibustion stimulation for 4 weeks on days 0 and 28 . In the metabolism measuring system (SCANET MV-10; MELQUEST Co., Ltd., Toyama, Japan) (Fig. 1), infrared rays are spread horizontally (lengthwise and crosswise) at 5-mm intervals, and the number of infrared rays blocked by the animal was counted (Kitaichi et al., 1994). The level of total locomotor activity in every 30-min interval was shown graphically over a 24hour period and this was used as the daily behavioral pattern. The daily level of locomotor activity was defined as the total locomotor activity over 24 hours. The level of locomotor activity in the daytime (9:00 AM -9:00 PM) and nighttime (9:00 PM-9:00 AM) was determined.

\section{Statistical analysis}

The data obtained in each group are expressed as mean \pm standard error. (SEM) The significance of differences between pairs of groups was assessed by the Wilcoxon sum rank test, and the level of significance was set at $P<0.05$.

\section{RESULTS}

Changes in body weight and serum T-chol level before and after moxibustion stimulation for 4 weeks

The body weight and serum T-chol level were significantly higher in the OVX-M and OVX-C groups than in the Normal group before and after moxibustion stimulation $(P<0.01$ each). These results suggest that the rats in the OVX-M and

Table 1. Body weight, serum total cholesterol level, tail surface temperature and level of locomotor activity before and after moxibustion simulation for 4 weeks. Results are shown as mean \pm SEM. ${ }^{*} P<0.05$; significantly different between normal and OVX-A, OVX-C. ${ }^{P}<<0.05$; significantly different between OVX-A and OVX-C.

\begin{tabular}{|c|c|c|c|c|c|c|c|c|}
\hline & \multicolumn{2}{|c|}{$\begin{array}{c}\text { Body weight } \\
(\mathrm{g})\end{array}$} & \multicolumn{2}{|c|}{$\begin{array}{l}\text { Serum T-Chol } \\
(\mathrm{mg} / \mathrm{dl})\end{array}$} & \multicolumn{2}{|c|}{$\begin{array}{c}\text { Tail surface tempera- } \\
\text { ture }\left({ }^{\circ} \mathrm{C}\right)\end{array}$} & \multicolumn{2}{|c|}{$\begin{array}{c}\text { Locomotor } \\
\text { activity (count) }\end{array}$} \\
\hline & Before & After & efore & After & Before & After & Before & \\
\hline & $88.4 \pm 3.7^{\text {*k }}$ & $246.3 \pm 4.5$ & 101 & $108.0 \pm 9.2^{* *}$ & $14.68 \pm 0.7$ & 15.88 & $\overline{9433 \pm 11372^{\star *}}$ & 133586 \\
\hline$C$ & $7.6 \pm 3.1^{* *}$ & $244.3 \pm 6.8^{* *}$ & $103.6 \pm 5.4^{* * *}$ & $112.6 \pm 13.2^{2-k}$ & $14.72 \pm 0.81^{*}$ & $14.47 \pm$ & $05923 \pm 12803^{k-k}$ & $104326 \pm 10825^{* *}$ \\
\hline Jormal & $164.6 \pm 1.8$ & $200.5 \pm 2.1$ & $80 . \overline{5 \pm 2.7}$ & $87.3 \pm 1.9$ & $16.03 \pm 0.27$ & $16.21 \pm 0.18$ & $148750 \pm 9425$ & $154383 \pm 19719$ \\
\hline
\end{tabular}


OVX-C groups tended to be obese.

Changes in tail surface temperature before and after moxibustion stimulation for 4 weeks

Three weeks after ovariectomy and before moxibustion stimulation, the tail surface temperature was significantly lower in the OVX-M and OVX-C groups than in the Normal group $(P<0.05, P<0.05)$. After moxibustion stimulation for 4 weeks, there was no significant difference in the tail surface temperature between the OVX-M group and Normal group $(P<0.05)$ and OVX-M group was higher than in the OVX-C group $(P<0.05)$ (Table 1 ).

Changes in locomotor activity before and after moxibustion stimulation for 4 weeks

In the Normal group, the pattern of locomotor activity was regular with a diphasic pattern throughout the study. In the OVX groups before moxibustion stimulation, there was no clear difference in the level of locomotor activity between the active and resting phases, and the pattern of locomotor activity was irregular. After moxibustion stimulation for 4 weeks in the OVX-M group, the pattern of locomotor activity had become diphasic with clear active and resting phases, similar to that observed in the Normal group (Fig. 2).

Moreover, before moxibustion stimulation, the daily level of locomotor activity was significantly lower in the OVX-M and OVX-C groups than in the Normal group $(P<0.01, P<0.01)$. After moxibustion stimulation for 4 weeks, the daily level of locomotor activity in the OVX-M group
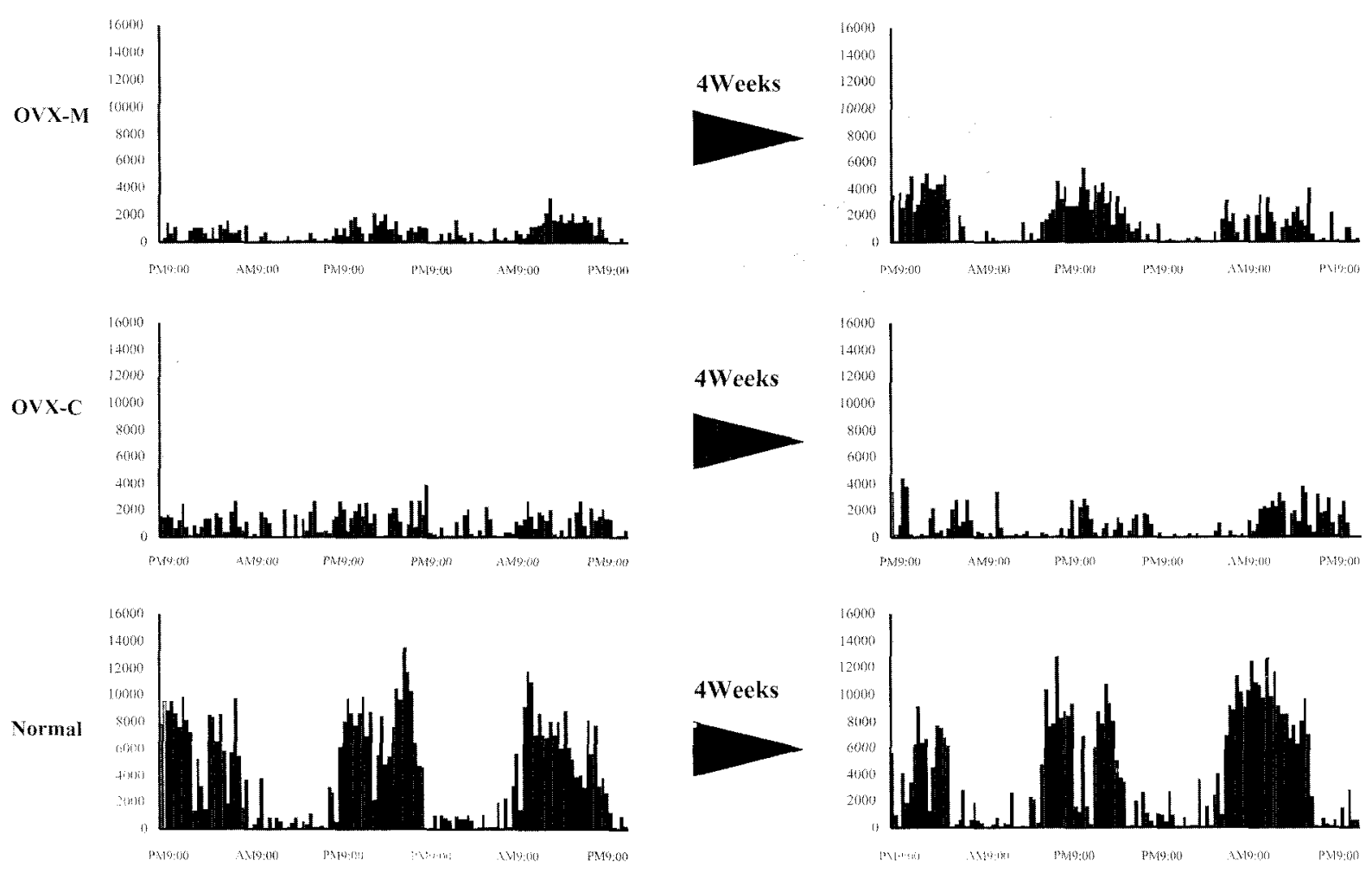

Fig. 2. Levels of locomotor activity in representative rats of the OVX-M, OVX-C and Normal groups before and after moxibustion stimulation for 4 weeks. The level of locomotor activity was measured three weeks after ovariectomy in the OVX-M and OVX-C rats before moxibustion stimulation. Only the OVX-M rats underwent moxibustion stimulation for 4 weeks. Each bar represents the number of counts in a 30 minute interval. 
was significantly higher than that in the OVX-C group $(P<0.05)$ (Table 1 ).

\section{DISCUSSION}

Many postmenopausal women in Japan go to moxibustion clinics to seek relief from menopausal symptoms. However, only a few studies on the effects of moxibustion about the symptoms of climacteric disturbance have been performed (Iwata, 2004).

The OVX rat has been widely studied and is used as a model of osteopenia since the secretion of estrogens is reduced due to ovariectomy. Estrogens induce the growth of reproductive organs as well as proliferation of the mammary glands, facilitate $\mathrm{Ca}$ deposition to bones, and reduce the serum $\mathrm{T}$ chol level. Therefore, estrogen deficiency induces a variety of symptoms (Yamaguchi et al., 1997).

In the present study, our finding that the body weight and serum level of $\mathrm{T}$-chol were increased in the OVX-M and OVX-C groups after ovariectomy may be taken as a sign of decreased secretion of estrogens (Ettinger et al., 1987).

Takeda et al. (2000) reported that the OVX rat could be used as a model of abnormal cold feelings because the tail surface temperature was lower than that in normal rats. In the present study, the pattern of locomotor activity of OVX rats that did not receive moxibustion stimulation was irregular.

These results demonstrate that OVX rats can be used as a model of climacteric disturbance. Since the tail surface temperature of OVX rats clearly increased after moxibustion stimulation for 4 weeks, moxibustion stimulation may improve the peripheral circulation. Further, it was confirmed using a metabolism measuring system that moxibustion stimulation for 4 weeks increased the level of locomotor activity. It was reported in a clinical study that moxibustion stimulation therapy relieved the pain of patients with frozen shoulder and low back pain (Kanai et al., 2000). Therefore, the increased locomotor activity of
OVX-M rats was presumably ascribable to the removal of blood circulation reduced blood flow in the peripheral circulation rather than the induction of stress by moxibustion.

We previously reported that administration of oriental medicine to postmenopausal women improved their menopausal symptoms (Kanai, 1998). In the present study, after moxibustion stimulation $\mathrm{CV}_{4}$ and $S P_{6}$ treated patients with climacteric disturbance, the pattern of irregular locomotor activity in the ovariectomized rats changed to a night-day diphasic pattern similar to that observed in the Normal rats, and the overall locomotor activity of the OVX-M rats significantly increased.

The possibility that moxibustion stimulation only affects the symptoms of climacteric disturbance cannot be ruled out.

We consider that it is important to provide moxibustion therapy in combination with western medicine for the treatment of climacteric disturbance. Further, we would like to perform extensive studies on the optimal time of moxibustion stimulation and the appropriate period of stimulation.

\section{REFERENCES}

Chen HC. (1987) Effects of moxibusting Point KuanYuan on cardiovascular and renal responses to histamine-induced shock. Am. J. Chinese Med. 15, $77-82$.

Ettinger B, Genant HK, Cann CE. (1987) Postmenopausal bone loss is prevented by treatment with lowdosage estrogen with calcium. Ann. Intern. Med. 106, $40-45$.

Harada K, Nakata Y. (1995) Effects of kampo therapy on serum estrogen levels and bone mineral content in climacteric disorder. Jpn. J. Orient. Med. 521-527.

Iwata J. (2004) A clinical case of acupuncture/ moxibustion therapy for climacteric disorders mainly headache. Osaka /. Clin. Acupuncture Moxibusiton 20, 83-85.

Jo ban N (2003) Acupuncture experimentable. Acupnincture Moxibustion 2, 94-96. 
Kanai S, Take A, Taniguchi N. (2000) Study of moxibusition for experimental arthritis model rat. Orient. Med. Pain Clin. 30, 16-21.

Kanai S. (1998) The effect of Kami-kihi-to on the maintenance of bone mass in patients with osteoporosis. J. Orient. Med. 49, 59-66.

Kitaichi K, Yamada K, Hasegawa T, Furukawa H, Nabeshima T. (1994) Effects of risperidone phencyclidine-induced behaviors comparison with haloperidol and ritanserin. Jpn. J. Phammacol. 66, 181189.

Takeda D, Kanai S, Taniguchi N, Umeda T. (2000) Experimental study on mechanizm of improvement by Kampo medicines for abnormal cold feelings against OVX (Ovarioectomy) rats. Kampo Newest Ther. 9, 259-262.

Uchida S, Suzuki A, Kagitani F, Nakajima K, Aikawa
Y. (2003) Effect of moxibustion stimulation of various skin areas on cortical cerebral blood flow in anesthetized rats. Am .J .Chinese Med. 31, 611-621.

Yamaguchi K, Yashita T, Kato M. (1997) Pharmacological effects of three products of chinese herbal remedies on experimental osteoporosis induced by ovariectomy in rats. Oyo Yakuri 57, 13-30.

Zhang W, Kanehara M, Ishida T, Guo Y, Wang X, Li G, Zhang B, Kondo H, Tachi S. (2004) Preventive and therapeutic effects of acupuncture on bone mass in osteopenic ovariectomized rats. Am. J. Chinese Med. 32, 427-443.

Zhao Ying-xia , Yan Zhen-guo, Wang Cai Yuan. (2000) The effects of acupuncture and moxibustion to BMD of rats after ovariectomy. Orient. Med. Pain Clin. 28, 107-110. 